\title{
Semiclassical matrix elements for a chaotic propagator in the Scar functions basis
}

\author{
Alejandro M.F Rivas \\ Departamento de Física, Comisión Nacional de Energía Atómica. Av. del Libertador 8250, \\ 1429 Buenos Aires, Argentina.
}

\begin{abstract}
A semiclassical approximation for the matrix elements of a quantum chaotic propagator in the scar function basis has been derived. The obtained expression is solely expressed in terms of canonical invariant objects. For our purpose, we have used, the recently developed, semiclassical matrix elements of the propagator in coherent states, together with the linearization of the flux in the neighborhood of a classically unstable periodic orbit of chaotic two dimensional systems. The expression here derived is successfully verified to be exact for a (linear) cat map, after the theory is adapted to a discrete phase space appropriate to a quantized torus.
\end{abstract}

PACS numbers: 03.65.Sq, 05.45.Mt 


\section{Introduction}

The Gutzwiller trace formula provides a tool for the semi-classical evaluation of the energy spectrum of a classically chaotic Hamiltonian system in terms of canonical invariants of periodic orbits (POs). However the number of long periodic orbits required to resolve the spectrum increases exponentially with the Heisenberg time $T_{H}$ [1]. For this reason, the approach is limited to eigenenergies close to the ground state [2]. Of course, Gutzwiller's formula is very attractive because it is given in terms of canonical invariants, and for this reason a lot of work has been dedicated to improve this theory [3]. In particular, the paper by Mehlig and Wilkinson [4], formulates the Guzwiller trace formula using coherent sates. This manifestly canonically invariant formulation allows to separate the contribution of each PO to the quantum evolution operator.

The semiclassical theory of short periodic orbits developed by Vergini and co-workers [5][10] is a formalism where the number of used POs increases only linearly with the mean energy density, allowing to obtain all the quantum information of a chaotic Hamiltonian system in terms of a very small number of short periodic orbits. The key elements in this theory are wave functions related to short unstable POs and then, it is crucial the evaluation of matrix elements between these wave functions.

In this context, these wave functions, named scar function because of its strong connection with the scarring phenomenon [11], are not restricted to a PO; it additionally includes dynamical information up to the Ehrenfest time and, as a result, is influenced by pieces of the stable and unstable manifolds near the PO. These wave functions define an optimal basis in chaotic systems [5, 12] and then, they have attracted an increasing interest in closed systems [13]. Moreover, they have been shown to be crucial for the understanding of long living resonances in open systems [14]-[16].

In recent work, estimates for the asymptotic behavior of off-diagonal matrix elements [9], and asymptotic expansions for matrix elements in the quantum cat maps [10] were derived. Also, recently a general semi-classical expression in phase space for the scar functions was obtained explicitly in terms of the classical invariants that generates the dynamics of the system [17].

In order to perform further developments to this theory of short periodic orbits, the semiclassical evaluation of matrix elements in a scar function basis set is an important objective. With these matrix elements at hand, the energy spectrum can be obtained without requiring an explicit computation of scar functions. This is the purpose of this paper. For our objective we use the recently developed semiclassical matrix elements of the quantum propagator between coherent states [18]. After what, we perform the needed time integrals to obtain the matrix elements of the propagator between scar functions.

Also, for two degrees of freedom Hamiltonian systems the dynamics is studied entirely within a surface of section transversal to the periodic orbit. That is, the full dynamics is studied through a two dimensional section map. With the purpose of showing the validity of our approximation, we have compared the general expression here founded with numerical calculations in a "realistic" system, the cat map, i.e. the quantization of linear symplectic maps on the torus. As was shown by Keating [19] in this case the semiclassical theory is exact, making this maps an ideal probe for our expression. After the formalism is adapted for a torus 
phase space, we see that the semiclassical expression here deduced coincide exactly with the numerically computed matrix elements for the cat maps.

Of course, in order to include nonlinear contributions a deeper understanding of the dynamics up to the Ehrenfest time is required; in this respect, enormous efforts were recently carried out in such a direction for the diagonal matrix elements 20].

This paper is organized as follows. In section 2 we introduce the definition of scar functions in terms of coherent states. Hence, we review the construction of the semiclassical matrix elements of the propagator in the coherent states basis [18]. We then show the utility of introducing the Weyl representation, and that our approach avoids for any complex trajectory.

Section 3 is devoted to obtain a semi classical expression for the matrix elements of the propagator in the scar function basis. For that purpose, we need to perform a linearization of the flux close to periodic orbits and to express the classical evolutions in the stable and unstable directions. Also, we obtain the expressions for a system with a discrete time evolution.

In Section 4 we study the particular case of the cat map where not only the semi-classical theory is exact but also the linear approximation is valid throughout the torus. After the semi classical expressions here deduced are adapted for a torus phase space, we see that they coincide exactly with the numerically computed matrix elements for the cat maps.

\section{Coherent states matrix elements}

Scar function states are the main object of study of the current work. According to [21]-[25], the scar function $\left|\varphi_{X}^{\phi}\right\rangle$ of parameter $\phi$ constructed on a single periodic point $X=(P, Q)$ is defined as

$$
\left|\varphi_{X}^{\phi}\right\rangle=\int_{-\infty}^{\infty} d t e^{i \phi t} f_{T}(t) \widehat{U}^{t}|X\rangle
$$

where $T=\ln \hbar$ is the Ehrenfest time, $|X\rangle$ is a coherent state centered in the point $X$ on the periodic orbit and $\widehat{U}^{t}$ is the unitary propagator that governs the quantum evolution of the system. While $f_{T}(t)$ is a decaying function that takes negligible values for $|t|>T / 2$. Without loss of generality, it will be convenient for our purpose to choose $f_{T}(t)=e^{-\left(\frac{4 t}{T}\right)^{2}}$, that is the scar function is

$$
\left|\varphi_{X}^{\phi}\right\rangle=\int_{-\infty}^{\infty} d t e^{i \phi t} e^{-\left(\frac{4 t}{T}\right)^{2}} \widehat{U}^{t}|X\rangle
$$

This wavefunctions have been shown, in the Husimi representation, to live in the neighborhood of the trajectory, resembling the hyperbolic structure of the phase space in their immediate vicinity [25], while its Wigner function also shows hyperbolic fringes asymptotic to the stable and unstable manifolds [17]. Wigner functions with hyperbolic structure have been spotted in previous works. For instance the pioneer work of Berry [26] shows this phenomenon for the spectral Wigner function in continuous systems while for maps this has been shown in [27]. While in the paper of Nicacio et al. 28] the hyperbolic fringes are observed for a superposition of two squeezed states with orthogonal squeezing directions. As is mention by Nicacio et al. [28] the scar functions are superpositions of Gaussian states with different degrees (and directions) of squeezing, i.e., they are generalized Gaussian cat states.

The purpose of this work, is to study semiclassically the matrix elements of the the unitary 
propagator in the scar function basis,

$$
\left\langle\varphi_{X_{1}}^{\phi_{1}}\left|\widehat{U}^{t}\right| \varphi_{X_{2}}^{\phi_{2}}\right\rangle
$$

From the definition of scar functions (11) we get,

$$
\left\langle\varphi_{X_{1}}^{\phi_{1}}\left|\widehat{U}^{t}\right| \varphi_{X_{2}}^{\phi_{2}}\right\rangle=\int_{-\infty}^{\infty} \int_{-\infty}^{\infty} d t_{1} d t_{2} e^{i\left(\phi_{2} t_{2}-\phi_{1} t_{1}\right)} f_{T}\left(t_{1}\right) f_{T}\left(t_{2}\right)\left\langle X_{1}\left|\widehat{U}^{\left(t+t_{2}-t_{1}\right)}\right| X_{2}\right\rangle
$$

Hence, we need to calculate $\left\langle X_{1}\left|\widehat{U}^{t}\right| X_{2}\right\rangle$ the matrix elements of the propagator in the coherent states basis. The semi-classical matrix elements of the propagator in coherent states basis have been obtained in [18], we will here reproduce the main steps of the procedure.

Let us write the propagator $\widehat{U}^{t}$ in terms of its, symplectically invariant, center or Weyl Wigner symbol $U^{t}(x)$ [29],

$$
\widehat{U}^{t}=\frac{1}{(\pi \hbar)^{L}} \int d x U^{t}(x) \widehat{R}_{x} \text { and } \quad U^{t}(x)=\operatorname{tr}\left[\widehat{R}_{x} \widehat{U}^{t}\right]
$$

where $\int d x$ is an integral over the whole phase space of $L$ degrees of freedom, while $\widehat{R}_{x}$ denotes the set of reflection operators thought points $x=(p, q)$ in phase space [29, 30] (see Appendix). Hence the coherent sates matrix elements can be written in terms of reflexions as

$$
\left\langle X_{1}\left|\widehat{U}^{t}\right| X_{2}\right\rangle=\left(\frac{1}{\pi \hbar}\right)^{L} \int\left\langle X_{1}\left|U^{t}(x) \widehat{R}_{x}\right| X_{2}\right\rangle d x .
$$

The coherent states on points $X=(P, Q)$ in phase space are obtained by translating to $X$ the ground state of the harmonic oscillator, its position representation is

$$
\langle q \mid X\rangle=\left(\frac{m \omega}{\pi \hbar}\right)^{\frac{1}{4}} \exp \left[-\frac{\omega}{2 \hbar}(q-Q)^{2}+i \frac{P}{\hbar}\left(q-\frac{Q}{2}\right)\right] .
$$

For simplicity, unit frequency $(\omega=1)$ and mass $(m=1)$ are chosen for the harmonic oscillator without loss of generality. The overlap of two coherent states is then

$$
\left\langle X \mid X^{\prime}\right\rangle=\exp \left[-\frac{\left(X-X^{\prime}\right)^{2}}{4 \hbar}-\frac{i}{2 \hbar} X \wedge X^{\prime}\right]
$$

with the wedge product

$$
X \wedge X^{\prime}=P Q^{\prime}-Q P^{\prime}=(\mathcal{J} X) \cdot X^{\prime} .
$$

The second equation also defines the symplectic matrix $\mathcal{J}$, that is

$$
\mathcal{J}=\left[\begin{array}{cc}
0 & -1 \\
1 & 0
\end{array}\right]
$$

As is shown in the Appendix the action of the reflection operator $\widehat{R}_{x}$ on a coherent state $|X\rangle$ is the $x$ reflected coherent state

$$
\widehat{R}_{x}|X\rangle=e^{\frac{i}{\hbar} X \wedge x}|2 x-X\rangle
$$


Inserting (9) and (77) in (5) the propagator in coherent states is obtained from the Weyl propagator

$$
\left\langle X_{1}\left|\widehat{U}^{t}\right| X_{2}\right\rangle=\frac{1}{(\pi \hbar)^{L}} e^{\frac{i X_{1} \wedge X_{2}}{2 \hbar}} \int U^{t}(x) e^{\left[\frac{i}{\hbar} x \wedge \xi_{0}-\frac{(\bar{X}-x)^{2}}{\hbar}\right]} d x
$$

with $\xi_{0} \equiv\left(X_{1}-X_{2}\right)$ the chord joining the points $X_{1}$ and $X_{2}$, while $\bar{X}=1 / 2\left(X_{1}+X_{2}\right)$ denotes their mid point.

Also, the semi-classical approximation for the propagator in the Weyl representation was performed in [29] so that

$$
U^{t}(x)_{S C}=\sum_{\gamma} \frac{2^{L} \exp \left\{i \hbar^{-1} S_{\gamma}^{t}(x)+i \frac{\pi}{2} \alpha_{\gamma}^{t}\right\}}{\left|\operatorname{det}\left(\mathcal{M}_{\gamma}^{t}+1\right)\right|^{\frac{1}{2}}}
$$

where the summation is over all the classical orbits $\gamma$ whose center lies on the point $x$ after having evolved a time $t[29]$. Then $S_{\gamma}^{t}(x)$ is the classical center generating function of the orbit, from which the chord $\xi$ joining the initial and final point of the orbit is obtained

$$
\xi=-\mathcal{J} \frac{\partial S_{\gamma}^{t}(x)}{\partial x}
$$

While $\mathcal{M}_{\gamma}^{t}=\frac{\partial^{2} S_{\gamma}^{t}(x)}{\partial x^{2}}$ stand for the monodromy matrix and $\alpha_{\gamma}^{t}$ its Morse index.

The metaplectic operators form a "double covering" of the symplectic matrices, since this property gives contributions to the Morse index [4]. If we follow the evolution of the symplectic matrix as the trajectory evolves, each time $\mathcal{M}_{\gamma}^{t}$ crosses a manifold where $\operatorname{det}\left(\mathcal{M}_{\gamma}^{t}+1\right)=0$ (caustic) the path contribution undergoes a divergence changing the sign from $-\infty$ to $\infty$. This change of the sign lets the quantum phase proceed by $\frac{\pi}{2}$. The Morse index $\alpha_{\gamma}^{t}$ therefore changes by \pm 1 when crossing caustics [26, 31].

For sufficiently short times such that the variational problem has an unique solution there will have a single chord. Although for longer times, there will be bifurcations producing more chords. In the case of a single orbit, the corresponding Morse index $\alpha_{\gamma}^{t}=0$.

The semiclassical approximation for the propagator (11) is inserted in (10) so that

$$
\left\langle X_{1}\left|\widehat{U}_{S C}^{t}\right| X_{2}\right\rangle=\left(\frac{2}{\pi \hbar}\right)^{L} e^{\frac{i}{2 \hbar} X_{1} \wedge X_{2}} \sum_{\gamma} e^{i \alpha_{\gamma}^{t}} \int \frac{\exp -\frac{1}{\hbar}(\bar{X}-x)^{2}}{\left|\operatorname{det}\left(\mathcal{M}_{\gamma}^{t}+1\right)\right|^{\frac{1}{2}}} \exp \frac{i}{\hbar}\left[S_{\gamma}^{t}(x)-\xi_{0} \wedge x\right] d x
$$

In order to perform the phase space integral in (13), it must be noted that classical orbits that start near $X_{2}$ and end near $X_{1}$ will have an important contribution in (13). These orbits have their center points close to $\bar{X}$. Hence, let us expand the center action up to quadratic terms near the mid point $\bar{X}$, so that,

$$
S_{\gamma}^{t}(x)=S_{\gamma}^{t}(\bar{X})+\bar{\xi} \wedge x^{\prime}+x^{\prime \dagger} B_{t} x^{\prime}+O\left(x^{\prime 3}\right)
$$

with $x=\bar{X}+x^{\prime}$ and $S_{\gamma}^{t}(\bar{X})$ is the action of the orbit through the point $\bar{X}$ for which the chord $\bar{\xi}$ is 


$$
\bar{\xi}=-\left.\mathcal{J} \frac{\partial S_{\gamma}^{t}(x)}{\partial x}\right|_{x=\bar{X}}
$$

while, the symmetric matrix $B_{t}$ is the Cayley representation of $\mathcal{M}_{\gamma}^{t}$

$$
\mathcal{J} B_{t}=\frac{1-\mathcal{M}_{\gamma}^{t}}{1+\mathcal{M}_{\gamma}^{t}}=\frac{1}{2} \frac{\partial^{2} S_{\gamma}^{t}(x)}{\partial x^{2}}
$$

Let us define the action $\widetilde{S}_{\gamma}^{t}(\bar{X})=S_{\gamma}^{t}(\bar{X})+\hbar \frac{\pi}{2} \alpha_{\gamma}^{t}$ in order to include the Morse index in the action. After the linearization of the flux around the middle point, expression (14) is inserted in (13) hence we get

$$
\left\langle X_{1}\left|\widehat{U}_{S C}^{t}\right| X_{2}\right\rangle=\left(\frac{2}{\pi \hbar}\right)^{L} \sum_{\gamma} \frac{\exp \frac{i}{\hbar}\left[\widetilde{S}_{\gamma}^{t}(\bar{X})-\xi_{0} \wedge \bar{X}+\frac{1}{2} X_{1} \wedge X_{2}\right]}{\left|\operatorname{det}\left(\mathcal{M}_{\gamma}^{t}+1\right)\right|^{\frac{1}{2}}} \times I
$$

with

$$
I=\int \exp \frac{1}{\hbar}\left[-x^{\prime \dagger} C x^{\prime}+i\left[x^{\prime \dagger} B_{t} x^{\prime}+\left(\bar{\xi}-\xi_{0}\right) \wedge x^{\prime}\right]\right] d x^{\prime}
$$

a quadratic integral. The matrix $C$ is the quadratic form that denotes the scalar product,

$$
x^{\prime 2}=x^{\prime} \cdot x^{\prime}=x^{\dagger} C x^{\prime},
$$

where $x^{\dagger}$ denotes the transposed vector. We now perform exactly the quadratic integral, using

$$
I=\int \exp \left[-\frac{1}{\hbar} x^{\prime \dagger} \mathcal{V}_{t} x^{\prime}+\frac{1}{\hbar} Y \cdot x^{\prime}\right] d x^{\prime}=\frac{(\pi \hbar)^{L}}{\sqrt{\left(\operatorname{det} \mathcal{V}_{t}\right)}} \exp \left[\frac{1}{4 \hbar} Y^{\dagger} \mathcal{V}_{t}^{-1} Y\right]
$$

From equation (16)

$$
\mathcal{V}_{t}=C-i B_{t}
$$

and

$$
Y=i \mathcal{J}\left(\bar{\xi}-\xi_{0}\right)=2 \mathrm{i} \mathcal{J} \delta_{\mathrm{t}},
$$

where $\bar{\xi}=x_{f}-x_{i}$ is the chord that joins $x_{f}$ and $x_{i}$, respectively the final and initial point of the orbit of center $\bar{X}$. This last expression defines the point shift $\delta_{t}$, so that

$$
\delta_{t}=\frac{1}{2}\left(\bar{\xi}-\xi_{0}\right)=x_{f}-X_{1}=X_{2}-x_{i}
$$

Note that, the point shift $\delta_{t}$ is zero if there is a classical orbit starting in the point $X_{2}$ and ending in $X_{1}$. Inserting (18) in (16), we get for the propagator in coherent states,

$$
\left\langle X_{1}\left|\widehat{U}_{S C}^{t}\right| X_{2}\right\rangle=2^{L} \sum_{\gamma} \frac{\exp \frac{i}{\hbar}\left[\widetilde{S}_{\gamma}^{t}(\bar{X})-\frac{1}{2} X_{1} \wedge X_{2}\right]}{\left[\operatorname{det} \mathcal{V}_{t}\left|\operatorname{det}\left(\mathcal{M}_{\gamma}^{t}+1\right)\right|\right]^{\frac{1}{2}}} \times \exp \left[\frac{-1}{\hbar} \delta_{t}^{\dagger} \widetilde{\mathcal{V}} \delta_{t}\right]
$$

with the complex matrix $\mathcal{V}_{t}$ and the point shift $\delta_{t}$ defined respectively in (19) and (21) while $\widetilde{\mathcal{V}}=\mathcal{J}^{\dagger} \mathcal{V}_{t}^{-1} \mathcal{J}$

In order to separate amplitude and phase terms in (22), it is useful to write 


$$
\widetilde{\mathcal{V}}=\mathcal{J}^{\dagger} \mathcal{V}_{t}^{-1} \mathcal{J}=\mathcal{J}^{\dagger} \frac{1}{C-i B_{t}} \mathcal{J}=\overline{\mathcal{C}}_{t}-i \overline{\mathcal{B}}_{t}
$$

with the real matrices

$$
\overline{\mathcal{C}}_{t}=\Re(\widetilde{\mathcal{V}}) \quad \text { and } \quad \overline{\mathcal{B}}_{t}=-\Im(\widetilde{\mathcal{V}})
$$

Also,

$$
\operatorname{det} \mathcal{V}_{t}=\left|\operatorname{det} \mathcal{V}_{t}\right| e^{i \varepsilon}
$$

with $\left|\operatorname{det} \mathcal{V}_{t}\right|$ denoting the modulus and $\varepsilon$ the argument.

Hence inserting (23) and (24) in the matrix elements of the coherent state propagator (22) we obtain

$$
\begin{array}{rlrl}
\left\langle X_{1}\left|\widehat{U}_{S C}^{t}\right| X_{2}\right\rangle & = & & 2^{L} \sum_{\gamma} \frac{1}{\left[\left|\operatorname{det} \mathcal{V}_{t}\right|\left|\operatorname{det}\left(\mathcal{M}_{\gamma}^{t}+1\right)\right|\right]^{\frac{1}{2}}} \exp \left[-\frac{\delta_{t}^{\dagger} \overline{\mathcal{C}}_{t} \delta_{t}}{\hbar}\right] \\
& \times & \exp \frac{i}{\hbar}\left[\widetilde{S}_{\gamma}^{t}(\bar{X})-\frac{1}{2} X_{1} \wedge X_{2}+\delta_{t}^{\dagger} \overline{\mathcal{B}}_{t} \delta_{t}+\hbar \frac{\varepsilon}{2}\right] .
\end{array}
$$

This last expression of the semiclassical matrix elements between two coherent states of the quantum propagator is entirely expressed in terms of real classical objects, namely the action $S_{\gamma}^{t}(\bar{X})$ of the classical real orbit whose mid point is $\bar{X}$, the point shift $\delta_{t}$, the monodromy matrix $\mathcal{M}_{\gamma}$ and its Cayley representation $B_{t}$ and $C$, the scalar product form. We must note that the phase term in the second line of the expression is clearly separated from the amplitude ones, in the first line. In this way it is important to remark the Gaussian term that dampens the amplitude for large values of the point shift $\delta_{t}$, that is for orbits centered on $\bar{X}$ that start far from the point $X_{2}$ (then end far from $X_{1}$ ). So as, the main contribution in the sum over classical orbits in (25) will come from the particular orbit $\gamma$, centered in $\bar{X}$, whose initial point $x_{i}$ lies the closest to $X_{2}$. Other orbits contributions will be highly damped by the exponential term involving the point shift $\delta_{t}$. Then, only this particular orbit will be taken into account in the next sections in order to calculate the matrix elements for scar functions.

It must be mentioned here that, extensive work has been previously performed for the coherent states matrix elements of the propagator. In particular, a complete semiclassical derivation was performed by Baranger et al. [32, while dos Santos and de Aguiar performed a Weyl ordering treatment in [33]. Although mathematically correct, both constructions involve an analytic continuation to complex trajectories, while expression (25), derived originally in [18], has the peculiarity to avoid complex trajectories, only the real canonical variables of the classical system are needed.

Also, note that, if $t=0$, the quantum propagator is just the identity operator in Hilbert space, the classical symplectic matrix is the identity, the center action is null, and so are the 
symmetric matrix $\left(B_{t=0}=0\right)$ and the chord $\bar{\xi}=2 \delta_{t}-X_{2}+X_{1}=0$. Hence

$$
\begin{aligned}
\left\langle X_{1}\left|\widehat{U}_{S C}^{0}\right| X_{2}\right\rangle & = & \left\langle X_{1} \mid X_{2}\right\rangle=2^{L} \frac{\exp \frac{i}{\hbar}\left[-\frac{1}{2} X_{1} \wedge X_{2}\right]}{|\operatorname{det}(2)|^{\frac{1}{2}}} \times \exp \left[-\frac{1}{4 \hbar}\left(X_{2}-X_{1}\right)^{2}\right] \\
& = & \exp \left[-\frac{\left(X_{2}-X_{1}\right)^{2}}{4 \hbar}-\frac{i}{2 \hbar} X_{1} \wedge X_{2}\right],
\end{aligned}
$$

and we recover the result (17) for the overlap of coherent sates. As we have seen in [18] expression (25) is exact for the case of linear systems.

\section{Matrix elements for Scar functions}

For the study of the matrix elements of the quantum propagator in the scar function basis, we must insert the expression for the matrix elements of the propagator in coherent sates basis (25) in the scar function matrix elements expression (3). In that way we get,

$$
\begin{aligned}
\left\langle\varphi_{X_{1}}^{\phi_{1}}\left|\widehat{U}_{S C}^{t}\right| \varphi_{X_{2}}^{\phi_{2}}\right\rangle & = & 2^{L} \exp \frac{i}{\hbar}\left[-\frac{1}{2} X_{1} \wedge X_{2}\right] \int_{-\infty}^{\infty} \int_{-\infty}^{\infty} d t_{1} d t_{2} e^{i\left(\phi_{2} t_{2}-\phi_{1} t_{1}\right)} f_{T}\left(t_{1}\right) f_{T}\left(t_{2}\right) \\
& \times & \frac{\exp \frac{i}{\hbar}\left[\widetilde{S}_{\gamma}^{t_{R}}(\bar{X})+\delta_{t_{R}}^{\dagger} \overline{\mathcal{B}}_{t_{R}} \delta_{t_{R}}+\hbar \frac{\varepsilon}{2}\right]}{\left[\left|\operatorname{det} \mathcal{V}_{t_{R}}\right|\left|\operatorname{det}\left(\mathcal{M}_{\gamma}^{t_{R}}+1\right)\right|\right]^{\frac{1}{2}}} \times \exp \left[-\frac{\delta_{t_{R}}^{\dagger} \overline{\mathcal{C}}_{t_{R}} \delta_{t_{R}}}{\hbar}\right],
\end{aligned}
$$

where $t_{R}=t+t_{2}-t_{1}$. Note that, in (26) only the contribution of a unique orbit is taken into account. As we have already mentioned the contributions of orbits with longer point shifts were neglected. With the choice for $f_{T}(t)$ made in (2) and performing the change of variables $t_{s}=t_{2}+t_{1}$ and $t_{r}=t_{1}-t_{2}$ it is possible to separate part of the time integrals so that,

$$
\begin{aligned}
& \left\langle\varphi_{X_{1}}^{\phi_{1}}\left|\widehat{U}_{S C}^{t}\right| \varphi_{X_{2}}^{\phi_{2}}\right\rangle=\exp \frac{i}{\hbar}\left[-\frac{1}{2} X_{1} \wedge X_{2}\right] \int_{-\infty}^{\infty} d t_{s} e^{\frac{i}{2}\left(\phi_{2}-\phi_{1}\right) t_{s}} e^{-2 t_{s}^{2} / T^{2}} \int_{-\infty}^{\infty} d t_{r} e^{-2 t_{r}^{2} / T^{2}} e^{-\frac{i}{2}\left(\phi_{2}+\phi_{1}\right) t_{r}} \\
& \times \quad \frac{\exp \frac{i}{\hbar}\left[\widetilde{S}_{\gamma}^{t-t_{r}}(\bar{X})+\delta_{t-t_{r}}^{\dagger} \overline{\mathcal{B}}_{t-t_{r}} \delta_{t-t_{r}}+\hbar \frac{\varepsilon}{2}\right]}{\left[\left|\operatorname{det} \mathcal{V}_{t-t_{r}}\right|\left|\operatorname{det}\left(\mathcal{M}_{\gamma}^{t-t_{r}}+1\right)\right|\right]^{\frac{1}{2}}} \times \exp \left[-\frac{\delta_{t-t_{r}}^{\dagger} \overline{\mathcal{C}}_{t-t_{r}} \delta_{t-t_{r}}}{\hbar}\right] .
\end{aligned}
$$

Performing the first time integral, defining $A=T \sqrt{\frac{\pi}{2}} e^{-\frac{T^{2}\left(\phi_{2}-\phi_{1}\right)^{2}}{32}}$, and changing the variables to $t^{\prime}=t-t_{r}$ we get:

$$
\begin{aligned}
\left\langle\varphi_{X_{1}}^{\phi_{1}}\left|\widehat{U}_{S C}^{t}\right| \varphi_{X_{2}}^{\phi_{2}}\right\rangle & = & A \exp \frac{i}{\hbar}\left[-\frac{1}{2} X_{1} \wedge X_{2}\right] \int_{-\infty}^{\infty} d t^{\prime} e^{-2\left(t-t^{\prime}\right)^{2} / T^{2}} e^{-\frac{i}{2}\left(\phi_{2}+\phi_{1}\right)\left(t-t^{\prime}\right)} \\
& \times & \frac{\exp \frac{i}{\hbar}\left[\widetilde{S}_{\gamma}^{t^{\prime}}(\bar{X})+\delta_{t^{\prime}}^{\dagger} \overline{\mathcal{B}}_{t^{\prime}} \delta_{t^{\prime}}+\hbar \frac{\varepsilon}{2}\right]}{\left[\left|\operatorname{det} \mathcal{V}_{t^{\prime}}\right| \operatorname{det}\left(\mathcal{M}_{\gamma}^{t^{\prime}}+1\right) \mid\right]^{\frac{1}{2}}} \times \exp \left[-\frac{\delta_{t^{\prime}}^{\dagger} \overline{\mathcal{C}}_{t^{\prime}} \delta_{t^{\prime}}}{\hbar}\right] .
\end{aligned}
$$

Equation (28) expresses the semicalssical approximation of the matrix elements of the quantum propagator in the scar function basis uniquely in terms of classical objects. However, 
at this point expression (28) has the inconvenient that we need the evaluation of each one of this classical objects, for all the times of integration involved. In what follows, we will obtain explicit expression for the classical objects involved in (28).

For that purpose, we will first perform the study on a surface of section that is transversal to the flux and passing through $\bar{X}$. In analogy with classical Poincare surfaces of section. The flux restricted to this section is now a map on the section, for this map the time is discrete and time integrals must be replaced by summations.

The study of autonomous fluxes through a map on surface of section is a standard procedure, in the case of billiards this is done through the well known Birkhoff coordinates. Also, quantum surface of section methods are shown to be exact [34 for general Hamiltonian systems.

For this procedure, we can choose coordinates near the periodic orbit of period $\tau$ where $X_{2}$ belongs, such that one coordinate is the energy $E$ and the conjugate coordinate is the time along the orbit. With this choice of coordinates, a point $x=(\tilde{x}, t, E)$ with now $\tilde{x}$ a $(2 L-2)$ vector on the so called central surface of section [29]. In order to perform our study on this surface of section near the fixed point $X_{2}$ we have linearized the flux in the the neighborhood of the orbit through $X_{2}$. That is, for the orbit $\gamma$ that starts in $x_{i}$ and end in $x_{f}: x_{f}=\mathcal{L}_{\gamma}^{t}\left(x_{i}\right) \approx \mathcal{M}_{\gamma}^{t} x_{i}$. Where $\mathcal{M}_{\gamma}^{t}$ is the symplectic matrix denoting this linearized time evolution. As was shown in [29], in the transformation $x_{f}=\mathcal{M}_{\gamma}^{t} x_{i}$ for times $t$ that are integer multiples of $\tau, t=n \tau$, the points $x_{f}=\left(\tilde{x}_{f}, t_{f}, E_{f}\right)$ and $x_{i}=\left(\tilde{x}_{i}, t_{i}, E_{i}\right)$ on the surface of section have the same energy $\left(E_{f}=E_{i}\right)$ and time along the orbit $\left(t_{f}=t_{i}\right)$ so we can write,

$$
\mathcal{M}_{\gamma}^{t}=\left(\begin{array}{c|c}
m_{\gamma}^{t} & 0 \\
\hline 0 & 10 \\
& 01
\end{array}\right)
$$

with

$$
\operatorname{det}\left[1+\mathcal{M}_{\gamma}^{t_{1}}\right]=4 \operatorname{det}\left[1+m_{\gamma}^{t_{1}}\right]
$$

where $m_{\gamma}^{t_{1}}$ is now the $(2 L-2) \times(2 L-2)$ symplectic matrix for the center map determined by the orbit $\gamma$ on the surface section, that is

$$
\tilde{x}_{f}=m_{\gamma}^{t} \tilde{x}_{i}
$$

From now on, the $2 L$ dimensional autonomous flux is studied through the $2 L-2$ map on the mentioned surface of section. Also the point $X_{2}$ on the periodic orbit of the flux is a fixed point for the map on the section.

For values of the time that are integer multiples of $\tau$ and for points $\tilde{x}$ on the surface of section, the expression (28) for the matrix elements of the propagator takes now the following form (replacing time integrations by summations):

$$
\begin{array}{rlrl}
\left\langle\varphi_{X_{1}}^{\phi_{1}}\left|\widehat{U}^{t}\right| \varphi_{X_{2}}^{\phi_{2}}\right\rangle & =\quad \exp \frac{i}{\hbar}\left[-\frac{1}{2} X_{1} \wedge X_{2}\right] A \sum_{n^{\prime}=-\infty}^{\infty} e^{-2\left(t-t^{\prime}\right)^{2} / T^{2}} e^{-\frac{i}{2}\left(\phi_{2}+\phi_{1}\right)\left(t-t^{\prime}\right)} \\
& \times \quad & \frac{\exp \frac{i}{\hbar}\left[\widetilde{S}_{\gamma}^{t^{\prime}}(\bar{X})+\delta_{t^{\prime}}^{\dagger} \overline{\mathcal{B}}_{t^{\prime}} \delta_{t^{\prime}}+\hbar \frac{\varepsilon}{2}\right]}{\left[\left|\operatorname{det} \mathcal{V}_{t^{\prime}}\right|\left|\operatorname{det}\left(\mathcal{M}_{\gamma}^{t^{\prime}}+1\right)\right|\right]^{\frac{1}{2}}} \times \exp \left[-\frac{\delta_{t^{\prime}}^{\dagger} \overline{\mathcal{C}}_{t^{\prime}} \delta_{t^{\prime}}}{\hbar}\right] .
\end{array}
$$


with $t=n \tau$ and $t^{\prime}=n^{\prime} \tau$ with $n$ and $n^{\prime}$ integer numbers. This last expression represents the semiclassical propagator matrix elements in scar function basis, on the surface of section that cuts transversally the periodic orbit on the point $X_{2}$. In order to deal with the infinite time summation in (31) we perform a cut off for values of $\left|t-t^{\prime}\right|$ greater that $T$, the Ehrenfest time, beyond which the time dependent Gaussian became negligible. Remember also the discussion according to the choice of the the function $f_{T}(t)$ in (1).

As we have already mentioned, we need to evaluate the classical objects involved in (31) to perform the time summation. Let us first, obtain expressions for the point shifts $\delta_{t^{\prime}}$ and the center action $\widetilde{S}_{\gamma}^{t}(\bar{X})$ in terms of the monodromy matrix. For that purpose, we linearize the flux in the the neighborhood of the fixed point $X_{2}$. That is,

$$
x_{f}=X_{1}+\delta_{t}=\mathcal{L}_{\gamma}^{t}\left(x_{i}\right)=\mathcal{L}_{\gamma}^{t}\left(X_{2}-\delta_{t}\right) \approx \mathcal{M}_{\gamma}^{t}\left(X_{2}\right)-\mathcal{M}_{\gamma}^{t} \delta_{t}=X_{2}-\mathcal{M}_{\gamma}^{t} \delta_{t},
$$

where $\mathcal{M}_{\gamma}^{t}$ is the symplectic matrix denoting this linearized time evolution in the neighborhood of $X_{2}$, the last equality hold because $X_{2}$ is a fixed point. Resolving for $\delta_{t}$ we get,

$$
\delta_{t}=-\frac{1}{\mathcal{M}_{\gamma}^{t}+1}\left(X_{1}-X_{2}\right)
$$

Equivalently we can perform the linearization using the center generating function near the point $X_{2}$, so that

$$
S_{\gamma}^{t}(x)=S_{\gamma}^{t}\left(X_{2}\right)+\left(x-X_{2}\right)^{\dagger} B_{t}\left(x-X_{2}\right)+O\left(x^{\prime 3}\right)
$$

Note that, the linear term $\xi_{2} \wedge\left(x-X_{2}\right)$ is not present here since $X_{2}$ is a fixed point, hence the chord $\xi_{2}$ passing thorough it is null, $\xi_{2}=-\left.\mathcal{J} \frac{\partial S_{\gamma}^{t}(x)}{\partial x}\right|_{X_{2}}=0$. Also, for $X_{2}$ being a fixed point

$$
S_{\gamma}^{t}\left(X_{2}\right)=t S_{X_{2}}
$$

where $S_{X_{2}}$ is the action of the periodic orbit in $X_{2}$ which the Morse index $\alpha_{\gamma}^{t}=t \alpha_{\gamma}$. Let us define the action $\widetilde{S}_{X_{2}}=S_{X_{2}}+\hbar \frac{\pi}{2} \alpha_{\gamma}$ in order to include the Morse index in the action.

The chord $\bar{\xi}$ of the orbit $\gamma$ centered in $\bar{X}$ is obtained by performing the derivative of the center generating function (32),

$$
\bar{\xi}=-\left.\mathcal{J} \frac{\partial S_{\gamma}^{t}(x)}{\partial x}\right|_{\bar{X}}=-2 \mathcal{J} B_{t}\left(\bar{X}-X_{2}\right)=-\mathcal{J} B_{t} \xi_{0} .
$$

So that (as was already seen):

$$
\delta_{t}=\frac{1}{2}\left(\bar{\xi}-\xi_{0}\right)=-\frac{1}{2}\left(\mathcal{J} B_{t}+1\right) \xi_{0}=-\frac{1}{\mathcal{M}_{\gamma}^{t}+1} \xi_{0}
$$

Hence, the center generating function in the middle point $\bar{X}$ is

$$
\widetilde{S}_{\gamma}^{t}(\bar{X})=t \widetilde{S}_{X_{2}}+\left(\bar{X}-X_{2}\right)^{\dagger} B_{t}\left(\bar{X}-X_{2}\right)=t \widetilde{S}_{X_{2}}+\frac{1}{4} \xi_{0}^{\dagger} B_{t} \xi_{0} .
$$

It is important to mention that the summation to be performed in (31) is a summation on the orbits $\gamma$ that starts near $X_{2}$ and after a time $t$ end up near $X_{1}$, having $\bar{X}$ as center point, 
this is a sum on the family of heteroclinic orbits as has been already seen in 9 . Inserting the expressions (33) and (34) respectively for the point shifts $\delta_{t}$ and the center action $\widetilde{S}_{\gamma}^{t}(\bar{X})$ in the scar function expressions (28), we obtain

$$
\begin{aligned}
\left\langle\varphi_{X_{1}}^{\phi_{1}}\left|\widehat{U}_{S C}^{t}\right| \varphi_{X_{2}}^{\phi_{2}}\right\rangle & =\quad A \exp \frac{i}{\hbar}\left[-\frac{1}{2} X_{1} \wedge X_{2}\right] \sum_{n^{\prime}=-\infty}^{\infty} e^{-2\left(t-t^{\prime}\right)^{2} / T^{2}} e^{-\frac{i}{2}\left(\phi_{2}+\phi_{1}\right)\left(t-t^{\prime}\right)} \\
& \times \quad \frac{\exp \frac{i}{\hbar}\left[t^{\prime} \widetilde{S}_{X_{2}}+\xi_{0}^{\dagger}\left(\frac{1}{4} B_{t^{\prime}}+D_{t^{\prime}}\right) \xi_{0}+\hbar \frac{\varepsilon}{2}\right]}{\left[\left|\operatorname{det} \mathcal{V}_{t^{\prime}}\right|\left|\operatorname{det}\left(\mathcal{M}_{\gamma}^{t^{\prime}}+1\right)\right|\right]^{\frac{1}{2}}} \exp \left[-\frac{\xi_{0}^{\dagger} E_{t^{\prime}} \xi_{0}}{\hbar}\right],
\end{aligned}
$$

with the matrices $E_{t}$ and $D_{t}$ so that

$$
E_{t}=\left(\frac{1}{\mathcal{M}_{\gamma}^{t}+1}\right)^{\dagger} \overline{\mathcal{C}}_{t}\left(\frac{1}{\mathcal{M}_{\gamma}^{t}+1}\right) \quad \text { and } \quad D_{t}=\left(\frac{1}{\mathcal{M}_{\gamma}^{t}+1}\right)^{\dagger} \overline{\mathcal{B}}_{t}\left(\frac{1}{\mathcal{M}_{\gamma}^{t}+1}\right)
$$

Equation (35) is a general expression only in term of classical objects, its difference from (31) is that we have used the linearization around the periodic orbit in order to express both the point shift and the center generating function only in terms of the monodromy matrix of the linearized transformation. In this way, the semiclassical approximation of the scar function's matrix elements involves uniquely the action of the classical orbit $\widetilde{S}_{X_{2}}$, the scalar product $C$ for the symplectic basis of vectors and the monodromy matrices $\mathcal{M}_{\gamma}^{t}$. From this former, we obtain it Cayley representation $B_{t}$ through equation (15), after what the complex matrix $\mathcal{V}_{t}$ is obtained with (19) and (24) expresses its exponential form, while the real matrices $\overline{\mathcal{C}}_{t}$ and $\overline{\mathcal{B}}_{t}$ defined in (23) allows to obtain $D_{t}$ and $E_{t}$ though (36).

Although, in order to perform the time summation we need the classical objects for all the different times involved. As we will show, for that purpose, it will be convenient to express them in the basis of eigenvectors of the symplectic matrix. For the case of a map with one degree of freedom (corresponding to a two degrees of freedom flux), this is the stable and unstable vector basis $\left(\vec{\zeta}_{u}, \vec{\zeta}_{s}\right)$ where the eigenvalues of the symplectic matrix $\mathcal{M}_{\gamma}^{t}$ are $\exp (-\lambda t)$ and $\exp (\lambda t)$, ( $\lambda$ is the stability or Lyapunov exponent of the orbit).

Let us then define $x_{s}$ and $x_{u}$ as canonical coordinates along the stable and unstable directions respectively such that $x=\left(x_{u}, x_{s}\right)=x_{u} \overrightarrow{\zeta_{u}}+x_{s} \overrightarrow{\zeta_{s}}$ with $\overrightarrow{\zeta_{u}} \wedge \overrightarrow{\zeta_{s}}=1$. As the basis formed by $\left(\vec{\zeta}_{u}, \vec{\zeta}_{s}\right)$ is non orthonormal, the scalar product of two vectors takes the form,

$$
x_{1} \cdot x_{2}=x_{1}^{\dagger} C x_{2}=\left[\zeta_{u}^{2} x_{1 u} x_{2 u}+\zeta_{s}^{2} x_{1 s} x_{1 s}+\vec{\zeta}_{u} \cdot \vec{\zeta}_{s}\left(x_{1 u} x_{2 s}+x_{1 s} x_{2 u}\right)\right] .
$$

That is, the scalar product matrix is,

$$
C=\left[\begin{array}{cc}
\zeta_{u}^{2} & \vec{\zeta}_{u} \cdot \vec{\zeta}_{s} \\
\vec{\zeta}_{u} \cdot \vec{\zeta}_{s} & \zeta_{s}^{2}
\end{array}\right]
$$

with $\zeta_{u}^{2}=\overrightarrow{\zeta_{u}} \cdot \vec{\zeta}_{u}$ and $\zeta_{s}^{2}=\vec{\zeta}_{s} \cdot \vec{\zeta}_{s}$. Since the transformation from the orthonormal basis $(\vec{i}, \vec{j})$ to the basis $\left(\vec{\zeta}_{u}, \vec{\zeta}_{s}\right)$ is symplectic

$$
\operatorname{det} C=\zeta_{u}^{2} \zeta_{s}^{2}-\left(\vec{\zeta}_{u} \cdot \vec{\zeta}_{s}\right)^{2}=1
$$


Also, in the $\left(\vec{\zeta}_{u}, \vec{\zeta}_{s}\right)$ basis,

$$
\mathcal{M}_{\gamma}^{t}+1=2 \cosh \left(\frac{\lambda t}{2}\right)\left[\begin{array}{cc}
e^{t \lambda / 2} & 0 \\
0 & e^{-t \lambda / 2}
\end{array}\right]
$$

hence

$$
\left|\operatorname{det}\left(\mathcal{M}_{\gamma}^{t}+1\right)\right|=4 \cosh ^{2}\left(\frac{\lambda t}{2}\right)
$$

is easily obtained only in terms of $\lambda$ and $t$. Analogously,

$$
\mathcal{M}_{\gamma}^{t}-1=2 \sinh \left(\frac{\lambda t}{2}\right)\left[\begin{array}{cc}
e^{t \lambda / 2} & 0 \\
0 & -e^{-t \lambda / 2}
\end{array}\right]
$$

while, $B_{t}$, the Cayley parametrization of $\mathcal{M}_{\gamma}^{t}$, is in this basis

$$
B_{t}=\left[\begin{array}{cc}
0 & \tanh (t \lambda / 2) \\
\tanh (t \lambda / 2) & 0
\end{array}\right]
$$

Hence, using the expression of the symmetric matrix $B_{t}$ (40) and the scalar product (37) we get the complex matrix

$$
\mathcal{V}_{t}=C-i B_{t}=\left[\begin{array}{cc}
\zeta_{u}^{2} & \vec{\zeta}_{u} \cdot \vec{\zeta}_{s}-i \tanh (t \lambda / 2) \\
\vec{\zeta}_{u} \cdot \vec{\zeta}_{s}-i \tanh (t \lambda / 2) & \zeta_{s}^{2}
\end{array}\right] .
$$

For which the complex determinant

$$
\operatorname{det} \mathcal{V}_{t}=\left[1+\tanh ^{2}(t \lambda / 2)+2 i \vec{\zeta}_{u} \cdot \vec{\zeta}_{s} \tanh (t \lambda / 2)\right]
$$

with modulus

$$
\left|\operatorname{det} \mathcal{V}_{t}\right|=\sqrt{\left(1+\tanh ^{2}(t \lambda / 2)\right)^{2}+\left(2 \vec{\zeta}_{u} \cdot \vec{\zeta}_{s} \tanh (t \lambda / 2)\right)^{2}}
$$

and argument

$$
\epsilon=\arctan \frac{2 \vec{\zeta}_{u} \cdot \vec{\zeta}_{s} \tanh (t \lambda / 2)}{1+\tanh ^{2}(t \lambda / 2)}
$$

can be explicitly written in terms of the time and the Lyapunov exponent. Now, inverting the matrix $\mathcal{V}_{t}$ (41) we get,

$$
\mathcal{V}_{t}^{-1}=\frac{1}{\operatorname{det} \mathcal{V}_{t}}\left[\begin{array}{cc}
\zeta_{s}^{2} & -\vec{\zeta}_{u} \cdot \vec{\zeta}_{s}+i \tanh (t \lambda / 2) \\
-\vec{\zeta}_{u} \cdot \vec{\zeta}_{s}+i \tanh (t \lambda / 2) & \zeta_{u}^{2}
\end{array}\right]=\frac{1}{\operatorname{det} \mathcal{V}_{t}}\left(C^{-1}+i B_{t}\right)
$$

Also, we must note that since the matrix $\mathcal{V}_{t}$ is symmetric, we get that

$$
\widetilde{\mathcal{V}}=\mathcal{J}^{\dagger} \mathcal{V}_{t}^{-1} \mathcal{J}=\frac{\mathcal{V}_{t}}{\operatorname{det} \mathcal{V}_{t}}=\frac{1}{\left|\operatorname{det} \mathcal{V}_{t}\right|^{2}}\left(\Re\left(\operatorname{det} \mathcal{V}_{t}\right)-i \Im\left(\operatorname{det} \mathcal{V}_{t}\right)\right)\left(C-i B_{t}\right)=\overline{\mathcal{C}}_{t}-i \overline{\mathcal{B}}_{t}
$$

Hence, in the stable and unstable vector basis $\left(\vec{\zeta}_{u}, \vec{\zeta}_{s}\right)$, the real matrices $\overline{\mathcal{C}}_{t}$ and $\overline{\mathcal{B}}_{t}$ take the form 


$$
\overline{\mathcal{C}}_{t}=\Re(\widetilde{\mathcal{V}})=\frac{1}{\left|\operatorname{det} \mathcal{V}_{t}\right|^{2}}\left[C\left(1+\tanh ^{2}(t \lambda / 2)\right)-2 B_{t} \vec{\zeta}_{u} \cdot \vec{\zeta}_{s} \tanh (t \lambda / 2)\right]
$$

and

$$
\overline{\mathcal{B}}_{t}=-\Im(\widetilde{\mathcal{V}})=\frac{1}{\left|\operatorname{det} \mathcal{V}_{t}\right|^{2}}\left[B_{t}\left(1+\tanh ^{2}(t \lambda / 2)\right)+2 C \vec{\zeta}_{u} \cdot \vec{\zeta}_{s} \tanh (t \lambda / 2)\right]
$$

with the symmetric matrix $B_{t}$, the scalar product matrix $C$ and the determinant det $\mathcal{V}_{t}$ respectively given by the expressions (40), (37) and (43). Inserting the expressions (38), (46) and (47) in the definition of the symmetric matrices $D_{t}$ and $E_{t}$ (36), we get

$$
D_{t}=-2 \frac{\tanh (t \lambda / 2)}{\operatorname{det}_{1}}\left[\begin{array}{cc}
-\zeta_{s}^{2}\left(\vec{\zeta}_{u} \cdot \vec{\zeta}_{s}\right) e^{-t \lambda} & 1+\tanh ^{2}(t \lambda / 2)+2\left(\vec{\zeta}_{u} \cdot \vec{\zeta}_{s}\right)^{2} \\
1+\tanh ^{2}(t \lambda / 2)+2\left(\vec{\zeta}_{u} \cdot \vec{\zeta}_{s}\right)^{2} & -\zeta_{u}^{2}\left(\vec{\zeta}_{u} \cdot \vec{\zeta}_{s}\right) e^{t \lambda}
\end{array}\right]
$$

and

$$
E_{t}=-\frac{1+\tanh ^{2}(t \lambda / 2)}{\operatorname{det}_{1}}\left[\begin{array}{cc}
-\zeta_{s}^{2} e^{-t \lambda / 2} & \frac{\overrightarrow{\zeta_{u}} \cdot \overrightarrow{\zeta_{s}}}{2 \sinh ^{2}(t \lambda / 2)+1} \\
\frac{\overrightarrow{\zeta_{u}} \cdot \overrightarrow{\zeta_{s}}}{2 \sinh ^{2}(t \lambda / 2)+1} & -\zeta_{u}^{2} e^{t \lambda / 2}
\end{array}\right]
$$

where we have defined

$$
\operatorname{det}_{1}=4 \cosh ^{2}(t \lambda / 2)\left|\operatorname{det} \mathcal{V}_{t}\right|^{2}
$$

It is important to note that, (48), (49), (40), (42) and (39) are respectively explicit expression of the symmetric matrices $D_{t}, E_{t}$ and $B_{t}$ and the determinants $\left|\operatorname{det} \mathcal{V}_{t}\right|$ and $\left|\operatorname{det}\left(\mathcal{M}_{\gamma}^{t}+1\right)\right|$ for any value of the time $t$. Inserting these expressions in (35), the time summation can be numerically performed. In this way, we obtain a semiclassical expression for the matrix elements of the propagator in the scar functions basis entirely in terms of classical features such as, the chord $\xi_{0}$ that joins the points $X_{2}$ and $X_{1}$, the action of the periodic orbit $\widetilde{S}_{X_{2}}$, the stable and unstable vectors $\vec{\zeta}_{u}, \vec{\zeta}_{s}$ and the Lyapunov exponent $\lambda$.

\section{Scar functions Matrix elements for the Cat Map}

Now the present theory is applied to the cat map i.e. the linear automorphism on the 2torus generated by the $2 \times 2$ symplectic matrix $\mathcal{M}$, that takes a point $x_{-}$to a point $x_{+}$: $x_{+}=\mathcal{M} x_{-} \bmod (1)$. In other words, there exists an integer 2-dimensional vector $\mathbf{m}$ such that $x_{+}=\mathcal{M} x_{-}-\mathbf{m}$. Equivalently, the map can also be studied in terms of the center generating function [36]. This is defined in terms of center points

$$
x \equiv \frac{x_{+}+x_{-}}{2}
$$

and chords

$$
\xi \equiv x_{+}-x_{-}=-\mathcal{J} \frac{\partial S(x, \mathbf{m})}{\partial x}
$$

where

$$
S(x, \mathbf{m})=x B x+x(B-\mathcal{J}) \mathbf{m}+\frac{1}{4} \mathbf{m}(B+\widetilde{\mathcal{J}}) \mathbf{m}
$$


is the center generating function. Here $B$ is a symmetric matrix (the Cayley parameterization of $\mathcal{M}$, as in (401) ), while

$$
\widetilde{\mathcal{J}}=\left[\begin{array}{l|l}
0 & 1 \\
\hline 1 & 0
\end{array}\right]
$$

We will study here the cat map with the symplectic matrix

$$
\mathcal{M}=\left[\begin{array}{ll}
2 & 3 \\
1 & 2
\end{array}\right] \text {, and symmetric matrix } B=\left[\begin{array}{cc}
-\frac{1}{3} & 0 \\
0 & 1
\end{array}\right] \text {. }
$$

This map is known to be chaotic, (ergodic and mixing) as all its periodic orbits are hyperbolic. The periodic points $x_{l}$ of integer period $l$ are labeled by the winding numbers $\mathbf{m}$, so that

$$
x_{l}=\left(\begin{array}{c}
p_{l} \\
q_{l}
\end{array}\right)=\left(\mathcal{M}^{l}-1\right)^{-1} \mathbf{m} .
$$

The first periodic points of the map are the fixed points at $(0,0)$ and $\left(\frac{1}{2}, \frac{1}{2}\right)$ and the periodic orbits of period 2 are $\left[\left(0, \frac{1}{2}\right),\left(\frac{1}{2}, 0\right)\right],\left[\left(\frac{1}{2}, \frac{1}{6}\right),\left(\frac{1}{2}, \frac{5}{6}\right)\right],\left[\left(0, \frac{1}{6}\right),\left(\frac{1}{2}, \frac{2}{6}\right)\right],\left[\left(0, \frac{5}{6}\right),\left(\frac{1}{2}, \frac{4}{6}\right)\right]$ and $\left[\left(0, \frac{2}{6}\right),\left(0, \frac{4}{6}\right)\right]$. The eigenvalues of $\mathcal{M}$ are $e^{-\lambda}$ and $e^{\lambda}$ with $\lambda=\ln (2+\sqrt{3}) \approx 1.317$. This is then the stability exponent for the fixed points, whereas the exponents must be doubled for orbits of period 2. All the eigenvectors have directions $\vec{\zeta}_{s}=\left(-\frac{\sqrt{3}}{2}, \frac{1}{2}\right)$ and $\vec{\zeta}_{u}=\left(1, \frac{1}{\sqrt{3}}\right)$ corresponding to the stable and unstable directions respectively.

Quantum mechanics on the torus, implies a finite Hilbert space of dimension $N=\frac{1}{2 \pi \hbar}$, and that positions and momenta are defined to have discrete values in a lattice of separation $\frac{1}{N}[35,30$. The cat map was originally quantized by Hannay and Berry [35] in the coordinate representation the propagator is:

$$
\left\langle\mathbf{q}_{k}\left|\hat{\mathbf{U}}_{\mathcal{M}}\right| \mathbf{q}_{j}\right\rangle=\left(\frac{i}{N}\right)^{\frac{1}{2}} \exp \left[\frac{i 2 \pi}{N}\left(k^{2}-j k+j^{2}\right)\right],
$$

where the states $\left\langle q \mid \mathbf{q}_{j}\right\rangle$ are periodic combs of Dirac delta distributions at positions $q=$ $j / N \bmod (1)$, with $j$ integer in $[0, N-1]$. In the Weyl representation [30], the quantum map has been obtained in [36] as

$$
\begin{aligned}
\mathbf{U}_{\mathcal{M}}(x) & =\frac{2}{|\operatorname{det}(\mathcal{M}+1)|^{\frac{1}{2}}} \sum_{\mathbf{m}} e^{i 2 \pi N[S(x, \mathbf{m})]} \\
& =\frac{2}{|\operatorname{det}(\mathcal{M}+1)|^{\frac{1}{2}}} \sum_{\mathbf{m}} e^{i 2 \pi N\left[x B x+x(B-\mathcal{J}) \mathbf{m}+\frac{1}{4} \mathbf{m}(B+\widetilde{\mathcal{J}}) \mathbf{m}\right]}
\end{aligned}
$$

where the center points are represented by $x=\left(\frac{a}{N}, \frac{b}{N}\right)$ with $a$ and $b$ integer numbers in $[0, N-1]$ for odd values of $N$ [30]. There exists an alternative definition of the torus Wigner function which also holds for even $N$.

The fact that the symplectic matrix $\mathcal{M}$ has equal diagonal elements implies in the time reversal symmetry and then the symmetric matrix $B$ has no off-diagonal elements. This property will be valid for all the powers of the map and, using (158), we can see that it implies in the quantum symmetry

$$
\mathbf{U}_{\mathcal{M}}^{l}(p, q)=\left(\mathbf{U}_{\mathcal{M}}^{l}(-p, q)\right)^{*}=\left(\mathbf{U}_{\mathcal{M}}^{l}(p,-q)\right)^{*} .
$$


for any integer value of $l$.

It has been shown [35] that the unitary propagator is periodic (nilpotent) in the sense that, for any value of $N$ there is an integer $k(N)$ such that

$$
\hat{\mathbf{U}}_{\mathcal{M}}^{k(N)}=e^{i \phi} .
$$

Hence the eigenvalues of the map lie on the $k(N)$ possible sites

$$
\left\{\exp \left[\frac{i(2 m \pi+\phi)}{k(N)}\right]\right\}, \quad 1 \leq m \leq k(N)
$$

For the cases where $k(N)\langle N$ there are degeneracies and the spectrum does not behave as expected for chaotic quantum systems. In spite of the peculiarities in this map, a very weak nonlinear perturbations of cat maps restores the universal behavior of non degenerate chaotic quantum systems spectra 37. Eckhardt [38] has argued that typically the eigenfunctions of cat maps are random.

The Scar Wigner Function on the torus depends on the definition of the periodic coherent state [39], with $\langle p\rangle=P$ and $\langle q\rangle=Q$. In accordance to (6)

$$
\left\langle\mathbf{X} \mid \mathbf{q}_{k}\right\rangle=\sum_{j=-\infty}^{\infty} \exp \left\{-\frac{1}{\hbar}\left[i P\left(j+\frac{Q}{2}-k / N\right)+\frac{1}{2}(j+Q-k / N)^{2}\right]\right\} .
$$

The Scar function is then defined on the torus as

$$
\left|\varphi_{\mathbf{X}, \phi}\right\rangle=\sum_{t=-\infty}^{\infty} e^{i \phi t} e^{-\left(\frac{4 t}{T}\right)^{2}} \mathbf{U}_{\mathcal{M}}^{t}|\mathbf{X}\rangle
$$

Remember that for maps, time only takes discrete values, then the time integral in (2) has been in this case replaced by a summation. Also, as we have already discuss, for our numerical computations we truncate the sum for times $|t|>T / 2$ where the Gaussian damping term became negligible.

In order to construct operators or functions on the torus we have to periodize the construction. This is done merely using the recipe [30] that for any operator its Weyl representation on the torus $\mathbf{A}(x)$ is obtained from is analogue in the plane $A(x)$ by

$$
\mathbf{A}(x)=\sum_{j=-\infty}^{\infty} \sum_{k=-\infty}^{\infty}(-1)^{2 j a+2 k b+j k N} A\left(x+\frac{(k, j)}{2}\right) .
$$

Indeed the construction on the torus from the plane is obtain in terms of averages over equivalent points, that are obtained by translation with integer chords: $\widehat{T}_{\vec{k}}$ where $\vec{k}=\left(k_{p}, k_{q}\right)$ is a two dimensional vector with integer components $k_{p}$ and $k_{q}$. Hence, the unit operator in the Hilbert space of the torus is 30

$$
\widehat{\mathbf{1}}_{\boldsymbol{N}}=\sum_{k=0}^{N-1}\left|\mathbf{q}_{k}\right\rangle\left\langle\mathbf{q}_{k}\right|=\left\langle\widehat{T}_{\vec{k}} e^{i 2 \pi\left(\chi \wedge \vec{k}+\frac{N}{4} \vec{k} \widetilde{\mathcal{J}} \vec{k}\right)}\right\rangle
$$

so that

$$
\left.\left.|\mathbf{X}\rangle=\widehat{\mathbf{1}}_{N}|X\rangle=\left\langle e^{i 2 \pi\left(\chi \wedge \vec{k}+\frac{N}{4} \vec{k} \widetilde{\mathcal{J}} \vec{k}\right)} \widehat{T}_{\vec{k}} \mid X\right\rangle\right\rangle=\left\langle e^{i 2 \pi\left(\chi \wedge \vec{k}+\frac{N}{4} \vec{k} \widetilde{\mathcal{J}} \vec{k}\right)} e^{-\frac{i}{2 \hbar} X \wedge \vec{k}} \mid X+\vec{k}\right\rangle\right\rangle
$$


In this way the coherent sates matrix elements for any operator on the torus are obtained through

$$
\left\langle\mathbf{X}_{1}|\widehat{\mathbf{A}}| \mathbf{X}_{2}\right\rangle=\left\langle e^{i 2 \pi\left(\chi \wedge \vec{k}+\frac{N}{4} \vec{k} \widetilde{J} \vec{k}\right)} e^{-\frac{i}{2 \hbar} X \wedge \vec{k}}\left\langle X_{1}|\widehat{A}| X_{2}+\vec{k}\right\rangle\right\rangle
$$

and analogously for the scar functions matrix elements.

In table 1 we compare, for different values of $N$ correspondingly $\hbar=1 /(2 \pi N)$, the exact Scar matrix elements for a cat map with the semi classical ones obtained with expression (35) taking in both cases the torus periodization (63). As we can observe the semi classical expression (35) is exact in this case. This fact is not surprising since the cat map is equivalent to a quadratic Hamiltonian system. Also, we have verified that, as was previously seen in [10], this matrix elements are no null only for values of $N$ that are multiple of four and in this cases the matrix elements $\left\langle\varphi_{X_{1}}^{\phi_{1}} \mid \varphi_{X_{2}}^{\phi_{2}}\right\rangle$ are real numbers.

\begin{tabular}{|c|c|c|c|c|}
\hline$N$ & \begin{tabular}{|c|c}
$\left\langle\varphi_{X_{1}}^{\phi_{1}}\right.$ & $\left.\varphi_{X_{2}}^{\phi_{2}}\right\rangle$
\end{tabular} & $\left.\begin{array}{|l|l}\left\langle\varphi_{X_{1}}^{\phi_{1}}\right. & \varphi_{X_{2}}^{\phi_{2}}\end{array}\right\rangle_{S C}$ & \begin{tabular}{|c|c|c|}
$\varphi_{X_{1}}^{\phi_{1}}$ & $\widehat{U}$ & $\varphi_{X_{2}}^{\phi_{2}}$
\end{tabular} & $\left.\begin{array}{|c|c|c}\varphi_{X_{1}}^{\phi_{1}} & \widehat{U} & \varphi_{X_{2}}^{\phi_{2}}\end{array}\right\rangle_{S C}$ \\
\hline 100 & 0.32170130 & 0.32170128 & $0.36448490+i 0.419063829$ & $0.36448489+i 0.419063830$ \\
\hline 101 & 0,0 & 0,0 & 0,0 & 0,0 \\
\hline 104 & 0.33082419 & 0.33082418 & $0.37520132+i 0.397240638$ & $0.37520133+i 0.397240637$ \\
\hline 200 & 0.36468529 & 0.36468530 & $0.45326651+i 0.358242569$ & $0.45326650+i 0.358242570$ \\
\hline
\end{tabular}

Table 1. Numerical comparison between the exact scar functions matrix elements and scar functions matrix elements obtained from expression (35). First column shows the different values of $N=1 /(2 \pi \hbar)$, the inverse of the Plank constant. Second column displays (real and imaginary parts) the exact of the matrix elements between the two scar functions constructed on the fixed points of the cat map. Third column shows the respective semi classical approximation, using expression (35), of the matrix elements shown in the second column. Fourth column displays (real and imaginary parts) the exact matrix elements between the two scar functions constructed on the fixed points now for one iteration of the quantum propagator for the cat map. Fifth column shows the respective semi classical approximation, using expression (35), of the matrix elements shown in the forth column.

\section{Discussion}

The semiclassical theory of short periodic orbits developed by Vergini and co-workers [5]-[10] is a formalism where the number of used periodic orbits needed to obtain the spectrum of a classically chaotic system increases only linearly with the mean energy density, allowing to obtain all the quantum information of a chaotic Hamiltonian system in terms of a very small number of short periodic orbits. The key elements in this theory are wave functions related to short unstable POs and then, it is crucial the evaluation of matrix elements between these wave functions.

In this work by means of the Weyl representation, we have obtained a semiclassical expression for this matrix elements of the propagator in scar functions basis entirely in terms of the classical canonical invariants such as, the chord that joins the points $X_{2}$ and $X_{1}$, the action of the periodic orbit, the stable and unstable vectors $\vec{\zeta}_{u}, \vec{\zeta}_{s}$ and the Lyapunov exponent $\lambda$. Also, the comparison with a system whose semiclassical limit is exact has allowed to correctly check the exactness of the obtained expression up to quadratic Hamiltonian systems. 
As has been already seen [10, 20, with these matrix elements at hand, the spectrum of the propagator can be obtained without requiring an explicit computation of scar functions. Of course, in order to include nonlinear contributions a deeper understanding of the dynamics up to the Ehrenfest time is required; in this respect, enormous efforts were recently carried out in such a direction for the diagonal matrix elements [20].

\section{Acknowledgments}

I am grateful to E. Vergini, M. Saraceno and G. Carlo for stimulating discussions and thanks the CONICET for financial support.

\section{Appendix: Reflection Operators in Phase Space}

Among the several representations of quantum mechanics, the Weyl-Wigner representation is the one that performs a decomposition of the operators that acts on the Hilbert space, on the basis formed by the set of unitary reflection operators. In this appendix we review the definition and some properties of this reflection operators.

First of all we construct the family of unitary operators

$$
\hat{T}_{q}=\exp \left(-i \hbar^{-1} q \cdot \hat{p}\right), \quad \hat{T}_{p}=\exp \left(i \hbar^{-1} p \cdot \hat{q}\right),
$$

and following [29], we define the operator corresponding to a general translation in phase space by $\xi=(p, q)$ as

$$
\begin{aligned}
\hat{T}_{\xi} & \equiv & \exp \left(\frac{i}{\hbar} \xi \wedge \hat{x}\right) \equiv \exp \left[\frac{i}{\hbar}(p \cdot \hat{q}-q \cdot \hat{p})\right] \\
& = & \hat{T}_{p} \hat{T}_{q} \exp \left[-\frac{i}{2 \hbar} p \cdot q\right]=\hat{T}_{q} \hat{T}_{p} \exp \left[\frac{i}{2 \hbar} p \cdot q\right],
\end{aligned}
$$

where naturally $\hat{x}=(\hat{p}, \hat{q})$. In other words, the order of $\hat{T}_{p}$ and $\hat{T}_{q}$ affects only the overall phase of the product, allowing us to define the translation as above. $\hat{T}_{\xi}$ is also known as a Heisenberg operator. Acting on the Hilbert space we have:

$$
\widehat{T}_{\xi}\left|q_{a}\right\rangle=e^{\frac{i}{\hbar} p\left(q_{a}+\frac{q}{2}\right)}\left|q_{a}+q\right\rangle
$$

and

$$
\widehat{T}_{\xi}\left|p_{a}\right\rangle=e^{-\frac{i}{\hbar} q\left(p_{a}+\frac{p}{2}\right)}\left|p_{a}+p\right\rangle .
$$

We, hence, verify their interpretation as translation operators in phase space. The group property is maintained within a phase factor:

$$
\hat{T}_{\xi_{2}} \hat{T}_{\xi_{1}}=\hat{T}_{\xi_{1}+\xi_{2}} \exp \left[\frac{-i}{2 \hbar} \xi_{1} \wedge \xi_{2}\right]=\hat{T}_{\xi_{1}+\xi_{2}} \exp \left[\frac{-i}{\hbar} D_{3}\left(\xi_{1}, \xi_{2}\right)\right]
$$

where $D_{3}$ is the symplectic area of the triangle determined by two of its sides. Evidently, the inverse of the unitary operator $\hat{T}_{\xi}^{-1}=\hat{T}_{\xi}^{\dagger}=\hat{T}_{-\xi}$. 
The set of operators corresponding to phase space reflections $\hat{R}_{x}$ about points $x=(p, q)$ in phase space, is formally defined in [29] as the Fourier transform of the translation (or Heisenberg) operators

$$
\widehat{R}_{x} \equiv(4 \pi \hbar)^{-L} \int d \xi \quad e^{\frac{i}{\hbar} x \wedge \xi} \widehat{T}_{\xi}
$$

Their action on the coordinate and momentum bases are

$$
\begin{array}{ll}
\hat{R}_{x}\left|q_{a}\right\rangle= & e^{2 i\left(q-q_{a}\right) p / \hbar}\left|2 q-q_{a}\right\rangle \\
\hat{R}_{x}\left|p_{a}\right\rangle= & e^{2 i\left(p-p_{a}\right) q / \hbar}\left|2 p-p_{a}\right\rangle,
\end{array}
$$

displaying the interpretation of these operators as reflections in phase space. Also, Using the coordinate representation of the coherent state (6) and the action of reflection on the coordinate basis (.8), we can see that the action of the reflection operator $\widehat{R}_{x}$ on a coherent state $|X\rangle$ is the $x$ reflected coherent state

$$
\widehat{R}_{x}|X\rangle=\exp \left(\frac{i}{\hbar} X \wedge x\right)|2 x-X\rangle .
$$

This family of operators have the property that they are a decomposition of the unity (completeness relation)

$$
\hat{1}=\frac{1}{2 \pi \hbar} \int d x \hat{R}_{x}
$$

and also they are orthogonal in the sense that

$$
\operatorname{Tr}\left[\hat{R}_{x_{1}} \hat{R}_{x_{2}}\right]=2 \pi \hbar \delta\left(x_{2}-x_{1}\right)
$$

Hence, an operator $\hat{A}$ can be decomposed in terms of reflection operators as follows

$$
\hat{A}=\frac{1}{2 \pi \hbar} \int d x A_{W}(x) \hat{R}_{x}
$$

With this decomposition, the operator $\hat{A}$ is mapped on a function $A_{W}(x)$ living in phase space, the so called Weyl-Wigner symbol of the operator. Using (.12) it is easy to show that $A_{W}(x)$ can be obtained by performing the following trace operation

$$
A_{W}(x)=\operatorname{Tr}\left[\hat{R}_{x} \hat{A}\right] .
$$

Of course, as it is shown in [29], the Weyl symbol also takes the usual expression in terms of matrix elements of $\hat{A}$ in coordinate representation

$$
A_{W}(x)=\int\left\langle q-\frac{Q}{2}|\hat{A}| q+\frac{Q}{2}\right\rangle \exp \left(-\frac{i}{\hbar} p Q\right) d Q .
$$

It was also shown in [29] that reflection and translation operators have the following composition properties

$$
\begin{aligned}
\widehat{R}_{x} \widehat{T}_{\xi} & =\widehat{R}_{x-\xi / 2} e^{-\frac{i}{\hbar} x \wedge \xi} \\
\widehat{T}_{\xi} \widehat{R}_{x} & =\widehat{R}_{x+\xi / 2} e^{-\frac{i}{\hbar} x \wedge \xi}
\end{aligned}
$$




$$
\widehat{R}_{x_{1}} \widehat{R}_{x_{2}}=\widehat{T}_{2\left(x_{2}-x_{1}\right)} e^{\frac{i}{\hbar} 2 x_{1} \wedge x_{2}}
$$

so that

$$
\widehat{R}_{x} \widehat{R}_{x}=\widehat{1} .
$$

Now using (16) and (15) we can compose three reflections so that

$$
\widehat{R}_{x_{2}} \widehat{R}_{x} \widehat{R}_{x_{1}}=e^{\frac{i}{\hbar} \Delta_{3}\left(x_{2}, x_{1}, x\right)} \widehat{R}_{x_{2}-x+x_{1}}
$$

where $\Delta_{3}\left(x_{2}, x_{1}, x\right)=2\left(x_{2}-x\right) \wedge\left(x_{1}-x\right)$ is the area of the oriented triangle whose sides are centered on the points $x_{2}, x_{1}$ and $x$ respectively.

\section{References}

[1] M.C.Gutzwiller, Chaos in Classical and Quantum Mechanics (Springer-Verlag, NY, 1990).

[2] J. P. Keating and M. Sieber, Proc. R. Soc. London, Ser. A, 447, 413 (1994).

[3] A. Voros, J. Phys. A: Math. Gen., 21, 685 (1988); M. V. Berry and J. P. Keating, J. Phys. A: Math. Gen. 23, 4839 (1990) ; P. Cvitanoviïc and B. Eckhardt, Phys. Rev. Lett., 63, 823 (1989);

P. Cvitanoviïc, P. E. Rosenqvist, G. Vattay G. and H. H. Rugh, Chaos, 3, 619 (1993).

[4] B. Mehlig and M. Wilkinson, Ann. Phys. (Leipzig) 10 6âĂŞ7, 541âĂŞ559 (2001).

[5] E. G. Vergini, J. Phys. A: Math. Gen., 33, 4709 (2000); E. G. Vergini and G. G. Carlo, J Phys. A: Math. Gen., 33, 4717 (2000).

[6] E.G. Vergini and G.G. Carlo, J. Phys. A: Math. Gen. 34, 4525 (2001).

[7] G.G. Carlo, E.G. Vergini and P. Lustemberg, J. Phys. A: Math. Gen. 35, 7965 (2002).

[8] D.A. Wisniacki, E.G. Vergini, R.M. Benito and F. Borondo, Phys. Rev. Lett.94, 054101 (2005).

[9] E.G. Vergini and D. Schneider, J. Phys. A: Math. Gen. 38, 587 (2005).

[10] E. G. Vergini, D. Schneider and A. M. F. Rivas, J. Phys. A: Math. Theor., 41, 405102 (2008).

[11] E. J. Heller, Phys. Rev. Lett., 53, 1515 (1984).

[12] L. Ermann and M. Saraceno, Phys. Rev. E, 78, 036221 (2008).

[13] S.-Y. Lee and S. C. Creagh, Ann. Phys., 307, 392 (2003); A. Vagov, H. Schomerus and V. V. Zalipaev, Phys. Rev. E, 80, 056202 (2009).

[14] M. Novaes, J. M. Pedrosa, D. Wisniacki, G. G. Carlo and J. P. Keating, Phys. Rev. E 80, 035202(R) (2009).

[15] L. A. Raviola, G. G. Carlo and A.M.F. Rivas, Phys. Rev. E, 81, 047201 (2010).

[16] L.A. Raviola, A.M.F. Rivas and G. G. Carlo, Physica D 240, 1818-1824 (2011).

[17] A.M.F. Rivas, J. Phys. A: Math. Gen. 40, 11057 (2007).

[18] A.M.F. Rivas http://lanl.arxiv.org/abs/1207.0012 arXiv:1207.0012v1 (2012).

[19] J. P. Keating, Nonlinearity 4, 309 (1991).

[20] E. G. Vergini, E. L. Sibert III, F. Revuelta, R. M. Benito and F. Borondo Euro. Phys Lett., 89 40013 (2010).

[21] G.G. de Polavieja, F. Borondo and R.M. Benito, Phys. Rev. Lett. 73, 1613 (1994).

[22] S. Nonnenmacher and A. Voros, J. Phys. A 30,295 (1997).

[23] L. Kaplan and E.J. Heller, Phys. Rev. E 59, 6609 (1999).

[24] D.A. Wisniacki, F. Borondo, E. Vergini and R.M. Benito Phys. Rev. E 63, 066220 (2001).

[25] F. Faure, S. Nonnenmacher and S. De Bièvre, Comm. in Math. Phys. 239(3) (2003).

[26] M. V. Berry, Proc. R. Soc. A 423, 219 (1989).

[27] A.M.F. Rivas and A.M. Ozorio de Almeida, Nonlinearity 15, 681 (2002).

[28] F. Nicacio, R.N.P. Maia, F. Toscano and R. O. Vallejos, Physics Letters A 374, 4385âĂŞ4392 (2010).

[29] A.M. Ozorio de Almeida, Physics Report 295, 266 (1998).

[30] A.M.F. Rivas and A.M. Ozorio de Almeida, Annals of Physics 276, 223 (1999).

[31] M.C. Gutzwiller in "Chaos and Quantum Physics", Les Houches Session LII, pg.205-248 Ed: M.-J. Giannonni, A. Voros and J. Zinn-Justin (1989). 
[32] M. Baranger et al, J. Phys. A: Math. Gen. 34, 7227 (2001).

[33] L.C. dos Santos and M.A.M. de Aguiar, J. Phys. A: Math. Gen. 39, 13465 (2006).

[34] T. Prosen, J. Phys. A: Math. Gen. 28 , 4133 (1995).

[35] J.H. Hannay and M.V. Berry, Physica D, 267 (1980).

[36] A.M.F. Rivas, M. Saraceno and A.M. Ozorio de Almeida, Nonlinearity 13 (2000) 341.

[37] M. Matos and A.M. Ozorio de Almeida, Annals of Physics 237, 46 (1995).

[38] B. Eckhardt, J. Phys. A: Math. Gen. 19, 1823 (1986).

[39] S. Nonnenmacher, Nonlinearity 10, 1569 (1997). 